\title{
ASSESSMENT OF WHEAT FOLIAR MYCOFLORA AND ITS MANAGEMENT STRATEGIES IN DISTRICT BHIMBER, AZAD KASHMIR, PAKISTAN
}

\author{
TANVEER, H. ${ }^{*}-$ ISHTIAQ, M. ${ }^{1}-$ MEHRBAN, A. ${ }^{2}-$ WAHEEDA, M. $^{1}-$ SHAHZAD, A. ${ }^{1}-$ \\ MEHWISH, M. $^{1}$ \\ ${ }^{I}$ Department of Botany, Mirpur University of Science and Technology Bhimber Campus, \\ Bhimber Azad Kashmir, Pakistan \\ ${ }^{2}$ Department of Chemistry, University of Gujrat, Pakistan \\ (phone: +92-3469642212) \\ *Corresponding author \\ e-mail: tanveerajk@gmail.com; drishtiaqajk@gmail.com \\ (Received 27 $7^{\text {th }}$ Apr 2016; accepted 22 $2^{\text {nd }}$ Jul 2016)
}

\begin{abstract}
In this research, an analysis of wheat foliar mycoflora was explored with detection of 21 foliar fungal species from eight cultivated varieties in the wheat fields of District Bhimber of Azad Kashmir, Pakistan. Out of 21 species, 19 were isolated from three wheat varieties viz: V1 (Fareed-2006), V3 (Lasani-2008) and V6 (Aas 2011). Wheat variety V8 (Galaxy-2013) showed less number (28.5\%) of fungal species invasion. Mycosphaerella graminicola was present ubiquitously on all eight wheat varieties with $100 \%$ prevalence while Cephalosporium gramineum was only found on three wheat varieties with $37.5 \%$. The minimum occurrence was shown by fungus Nigrospora sphaerica (15\%). In sub-division Samahni, wheat variety Fareed-2006 (V1) depicted the highest disease susceptibility with incidence of $63.3 \%$. The variety Galaxy-2013 was found the best crop in Samahni having least incidence value of $22.3 \%$. In sub-division Bhimber, wheat variety Seher-2006 was the most affected by mycoflora having highest disease incidence (60.0\%) while least infection was measured in Galaxy-2013 (20.9\%), being appropriate varietal crop for the area. In Barnala sub-division, Fareed-2006 indicated highest disease incidence $(59.7 \%$ ) while minimum disease incidence was measured in Galaxy-2013 (29.7\%). As general conclusion Galaxy-2013 was proved as the best crop variety in the study area being nonth or least infected by fungal taxa. In second aspect of experiment comprising of optimization of management and control strategies, the parameter of grain yield was measured. As a general without any pre-treatment of seed crop, the variety V8 was the best of with yield of $1543 \mathrm{~kg} / \mathrm{ha}$, that might be due to its genetic resistance or better eco-climatic adaptability. Out of applied management strategies; use of fungicides (Quadris and Headline) spray on wheat leaves proved to be better having rise of yield i.e., $1550 \mathrm{~kg} / \mathrm{ha}$ and $1560 \mathrm{~kg} / \mathrm{ha}$, respectively. The other strategy: use of biological products i.e. different plant extracts (Acacia nilotica, Azadirachta indica, Curcuma longa, Eucaylptus citriodora, Ficus bengalensis) spray proved that landmark rise was obtained in yield from variety V8 with $1739 \mathrm{~kg} / \mathrm{ha}$ as compared to others. This rise was huge $(1739 \mathrm{~kg} / \mathrm{ha})$ in comparison to the without treatment crop having $1543 \mathrm{~kg} / \mathrm{ha}$ yield and it was proved pre-treatment produces better crop than control (blank) sample culminating the result that use of bio-products (plant extracts) are the best for control and management of mycoflora of wheat.

Keywords: mycoflora, wheat varieties, foliar diseases, fungicides, biocontrol
\end{abstract}

\section{Introduction}

Wheat (Triticum aestivum L.) is the largest crop present worldwide, which occupies about $217 \mathrm{~m} /$ hectors $(\mathrm{FAO}, 2012)$ and among cereal crops it is ranked third with annual production about 651 million tons in year 2010. It is likely that eco-climatic and frequently environmental changes have bad impacts on wheat yields and quality (Ortiz et al., 2008; Bender and Weigel, 2011). Climate change influenced wheat production. Therefore, better 
methods are applied for disease management in near future (Ewert, 2012). Wheat has high relation to environmental and climatic conditions (Rosada et al., 2010). Excessive moisture in the field and storage, humidity, drought and temperature extremes are principal environmental factors that determine the intensity of mycotoxins contaminations (Coulombe, 1993).

This crop is also very sensitive to pests, especially pathogenic fungi that cause leaf, head, and stem diseases (Jaczewska, 2010). These diseases contribute annually to a significant loss in wheat production (Korbas, 2004). A comprehensive survey of wheat pathogens including viruses, fungi and bacteria was carried out by Bockus et al., (2010). It is estimated that $10 \%$ of net wheat yield losses were due to fungal and bacterial pathogens and only $2 \%$ yield was reduced by viruses (Oerke, 2006). The question arises that whether these overall estimated yield reduction will be higher or lower under globally fluctuating weather which will depend on the direct effects on different pathogens and the indirect effects through the host-plant interaction.

Current research shows fungal diseases because these are major components of yield losses in wheat crop at local level as well as worldwide. On the other hand, viral and bacterial pathogens are usually less important according to disease severity (Oerke, 2006). Another reason for this study is the extremely limited literature on viral and particularly bacterial wheat diseases with respect to climate change (Juroszek and von Tiedemann, 2011).

Changes of different wheat pathogens in India and Pakistan is a future risk (Kaur et al., 2008). For example, the importance of stripe rust and Kernel bunt in Punjab, India is assumed to be reduced in the future due to temperature and humidity changes. On the other hand, the importance of leaf rust, foliar blights, Fusarium head blight, and stem rust may increase in Punjab in the future, the latter disease particularly in the absence of resistance in wheat cultivars (Kaur et al., 2008).

Wheat varieties are affected by different diseases which reduce quantitative as well as qualitative yield losses in Pakistan. The foliar diseases like leaf and glumes blotch, powdery mildew, three types of wheat crop rusts, tan spot, head blight and smuts are the quickest spreading diseases in different wheat varieties. Yield losses reach upto 30\% and depend on yield frequency according to environmental as well as wheat cultivar conditions.

Stripe rust is a more serious fungal disease of wheat crop. It infects susceptible host cultivars with symptoms occurring about one week after initial infection occurs. The disease often causes severe grain yield and quality loss as stripe rust can appear and spread rapidly when climatic conditions favor the disease. Stripe rust is reported in over 60 countries worldwide.

Fusarium culmorum, Fusarium graminearum and Fusarium sporotrichioides are the main producers of trichothecenes. These toxins are common fungal contaminants of cereals and occur naturally worldwide in the cultivation of wheat and other cereals (Brown et al., 2001; Champeil et al., 2004; Wagacha and Muthomi, 2007).

Therefore, management of fungal diseases is necessary for a sustainable agriculture. Crop management practices affect the development of the disease epidemics. In the humid subtropics of South Asia, there is evidence of stress conditions, which favor foliar blight (Dubin and Bimb, 1994). Factors such as minimum tillage or surface seeding, irrigation, late planting, or low soil fertility may be responsible for higher foliar blight severity in the wheatbased cropping systems of the Indo-Gangetic plains (Sharma and Duveiller, 2003). Lower disease severity with higher nitrogen application was reported by Chaurasia and Duveiller (2006) and Sharma and Duveiller (2004). However, Singh et al., (1998) reported more disease infection on higher nitrogen application. Selection and breeding for resistant cultivars is the 
main disease management strategy. Planting resistant cultivars is one of the least expensive and most effective management strategies to prevent diseases.

Fungicides are widely used to manage foliar wheat diseases in several countries of world (Carmona et al., 1999). The response to fungicide application depends on the severity of specific foliar diseases, cultivar disease resistance or tolerance, management practices and environmental conditions (Roth and Marshall, 1987; Varga et al., 2005; Carignano et al., 2008). Fungicides applied at flag leaf and spike emergence of winter wheat increased mean grain weight and grain yield when they extended canopy life (Gooding, 2006). Triazoles and Strobilurins are the most common systemic fungicides used to control foliar diseases on wheat. These types of fungicides generally move upward in the transpiration stream and may accumulate at the leaf margins (Arregui and Puricelli, 2008).

\section{Introduction of the study area}

The study area viz: District Bhimber (Figure 1) consists of three sub-divisions (tehsils). The environmental conditions of this district are ubiquitous in three tehsils. Geographcally, the district is located between latitude $32-48^{\circ}$ to $33-34^{\circ}$ and longitude $73-$ $55^{\circ}$ to $74-45^{\circ}$ and has an area of $1516 \mathrm{~km}^{2}$. The climate of this area is constant. Hot summer temperature is often over $38-46^{\circ} \mathrm{C}$ from May to September and cold winters (4$10^{\circ} \mathrm{C}$ ). The total average rain fall is about $1233 \mathrm{~mm}$ per month. Hail occurs occasionally during the month of February and March. Humidity remains high in rainy season and winters. Topographically, the area is semi mountainous and plain. The southern part is plain while northern part consists of rough and precipitates steep sand stone hills and rugge. The plain areas of district Bhimber are very fertile for crops cultivation.

The objective of this research was to survey foliar diseases associated with wheat varieties; to measure the incidence, prevalence and severity of foliar diseases of selected eight wheat varieties; to improve wheat yields by reducing or eradicate foliar fungal diseases through fungicides treatments and biological treatment by use of plant extracts.

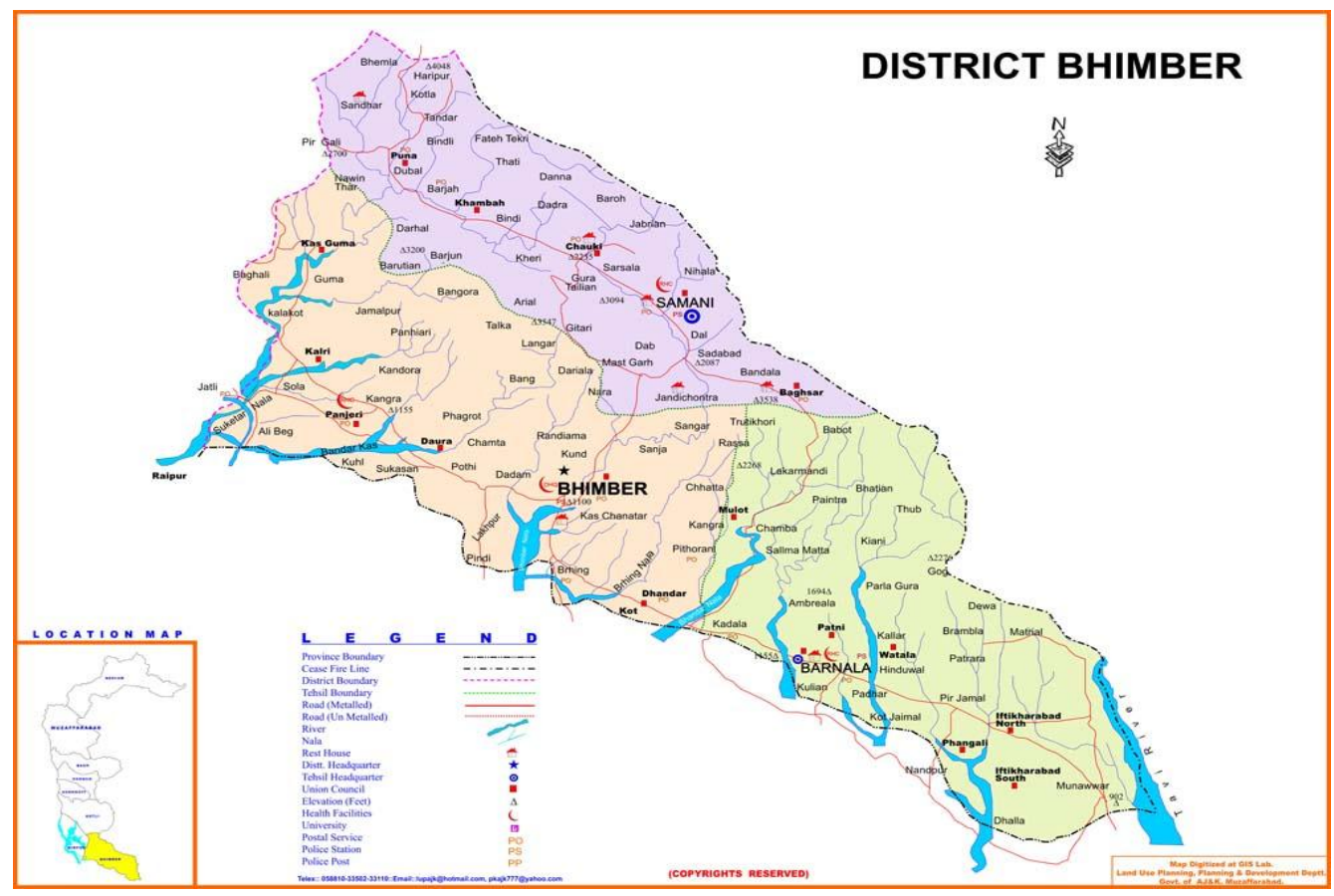

Figure 1. Map of the study area (District Bhimber) 


\section{Materials and Methods}

\section{Sample collection}

In this research study, all wheat growing areas of the district were surveyed. Fungal affected wheat leaves were collected in plastic bags and stored at room temperature for assessment and analyses of fungal diseases. The leaves were collected at seedling, young and mature stages. Morphological symptoms were recorded for presence or absence of foliar diseases in wheat growing areas of district Bhimber Azad Jammu and Kashmir (AJ\&K). Leaves of four common plants were collected for biological treatments against different fungi. The disease free and fresh plants were selected for this investigation. About two gram $(02 \mathrm{~g})$ of fresh and healthy leaves were taken for each solvent. Then, surface sterilized with $1 \%$ Sodium hypochlorite and alcohol for 2-3 minutes and the plant materials were washed thoroughly with distilled water.

The following methods were applied for assessment of foliar diseases.

\section{Assessment of disease prevalence by diagonal transect method}

Affected leaves of wheat varieties were collected from different field trails from three tehsils. The samples were collected from five points along with a diagonal transect as X-pattern (Anonymous, 2003). At each sampling site, the collected foliar samples were put in sterile flasks and transferred to laboratory for further fungal analysis, identification and purification (Iftikhar et al., 2012). The prevalence of disease was measured according to presence or absence of foliar spots in each field trail. The prevalence and severity (\% age) was calculated by applying following formulae:

$$
\text { i. DiseasePrevalece }(\%)=\frac{\text { Locations with filiar disease }}{\text { Total locations }} \times 100
$$

$$
\text { ii. Severity }=\frac{\sum(\mathrm{a} \times \mathrm{b})}{\mathrm{n} \times \mathrm{z}} \times 100
$$

where $\sum(\mathrm{a} \times \mathrm{b})=$ Sum of the symptomatic plants and their corresponding scoring scales, $\mathrm{n}=$ Total number of sampled plants and $\mathrm{z}=$ Highest Score Scale.

The assessment of the disease was made in double digit system which comprises of two digits representing the vertical disease progress and percentage of the blight area covered (severity). The first digit gives the relative height of the disease in 0-9 scale as proposed by Saari and Prescot (1985) and second digit represents the percentage area covered by the blight pathogen on the flag leaf and one below it which is as under:

$10 \%=1,20 \%=2,30 \%=3,40 \%=4,50 \%=5,60 \%=6,70 \%=7,80 \%=8,90 \%=9$

As for example the disease score of 55 denotes that the first digit of 5 represents the height of the disease in' 0-9 scale whereas the second digit exhibits average disease severity in percent on the flag leaf and one below it.

\section{Assessment of disease incidence (diagonal transect method)}

A fixed plot survey was applied for wheat leaves collection. The plants within the fixed plot were assessed for foliar diseases and leaf samples were collected from 
selected localities by X pattern (Sharma and Duveiller, 2003) and brought to laboratory for further fungal study (Iftikhar et al., 2012). Five plants were selected by diagonal transect method for documentation of incidence of different types of foliar diseases (Teng and James, 2001) by using following formula:

$$
\text { iii. Incidence }(\%)=\frac{\text { Number of leaves showing foliar spots }}{\text { Total No. of leaves of sample }} \times 100
$$

\section{Identification of fungi by agar plate method}

In agar plate method (APM), collected samples were placed on sterile PDA media. The PDA media prepared by mixing of prepared PDA media in $1000 \mathrm{ml}$ distilled water. The media autoclaved at fifteen (15) psi pressure for twenty (20) minutes in autoclave. $15 \mathrm{ml}$ media transferred in each petridish under aseptic circumstances. Foliar samples were surface sterilized in $1 \% \mathrm{NaOCl}_{2}$ for three to five minutes and then given three washing treatments with distilled water. The effected parts were transferred on PDA media after distillation. After 3-5 days, fungi were detected and identified by morphological characteristics (Domsch et al., 1980, Hussain et al., 2011).

Isolated fungi were purified by sub-culturing on PDA media (Usmani and Ghaffar, 1982). The plates were incubated at room temperature for 3-5 days. After the complete growth of fungi, the fungal cultures were sealed and preserved in refrigerator for future use.

\section{Disease management strategies}

There are different management strategies were applied to control foliar diseases of wheat varieties in district Bhimber Azad Kashmir.

\section{Fungicide treatments (chemical control)}

In field trails, each wheat variety was sprayed with five fungicides i.e., Tilt $(0.29$ $\left.1 \mathrm{ha}^{-1}\right)$, Proline $\left(0.761 \mathrm{ha}^{-1}\right)$, Strobilurins $\left(0.89 \mathrm{l} \mathrm{ha}^{-1}\right)$, Quadris $\left(0.581 \mathrm{ha}^{-1}\right)$ and Headline at different rates. The fungicides were applied on leaves of each variety in selected fields separately. The timing of different fungicides was different for better result. After all treatments, wait for fruit ripe and harvested the crop and calculated the yield of each wheat variety (Fakir, 1999).

\section{Biological treatments (biocontrol)}

As fungicides were sprayed, similarly different plant extracts were sprayed on all selected eight wheat varieties for reduction of fungal contaminations and better yields. This treatment method is more reliable as compared to fungicides because this method has few/no side effects Yield (kg/ha) and 1000-grain weight were measured after harvesting all plots (Pathak and Razia, 2013).

\section{Statistical analysis}

The data were evaluated by help of computer software MSTAT-C. Some data was analyzed statistically by LSD and ANOVA. Significance was calculated at $p<0.05$ and $p<0.01$ levels of probability. Each value is mean of three replicates (Steel et al., 1996; Pathak and Razia, 2013). 


\section{Results}

Wheat (Triticum aestivum) is being attacked by several fungal diseases many of them are foliar pathogens. In this research, 21 different foliar fungal species from 40 foliar samples of eight wheat varieties were collected from three tehsils (sub-divisions) of district Bhimber Azad Jammu and Kashmir during the year 2014 and 2015 for mycofloral analysis (Table 1). The foliar samples of eight wheat varieties were collected and preserved in Laboratory of Botany, Mirpur University of Science and Technology (MUST), Bhimber Campus Azad Jammu and Kashmir for further experimental works.

Highest number of fungal species were identified from wheat variety V1 (Fareed2006), V3 (Lasani-2008) and V6 (Aas 2011). Out of 21 fungal species 19 (90.5\%) appeared on these three wheat varieties. Less number of fungal species i.e., $6(28.5 \%)$ were isolated from wheat variety V8 (Galaxy-2013). In the current analysis, occurrence of fungal species was found variable; Mycosphaerella graminicola was present on all wheat varieties $(100 \%)$ while Cephalosporium gramineum was only found on three wheat varieties with $37.5 \%$ as indicated in Table 1 . The identification of fungal pathogens was presented by field photography. Figure 2 shows the attack of Puccinia tritici-repentis, Figure 3 the attack of pathogen Stagonospora nodorum. Similarly, Figure 4 presents attack of Puccinia striiformis on wheat crop, Figure 5 the attack of pathogen Drechslera tetramera, while Figure 6 shows attack of various fungal species on wheat crop and Figure 7 the presence of Alternaria species on wheat.

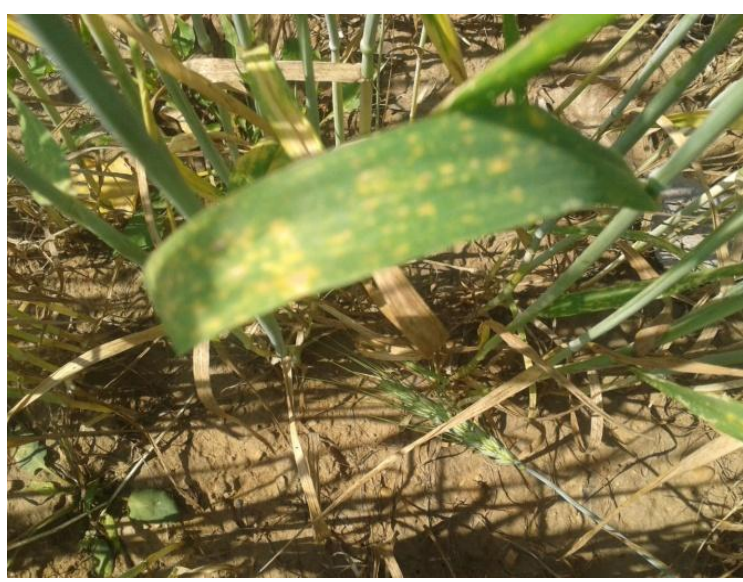

Figure 2. Attack of Puccinia tritici-repentis

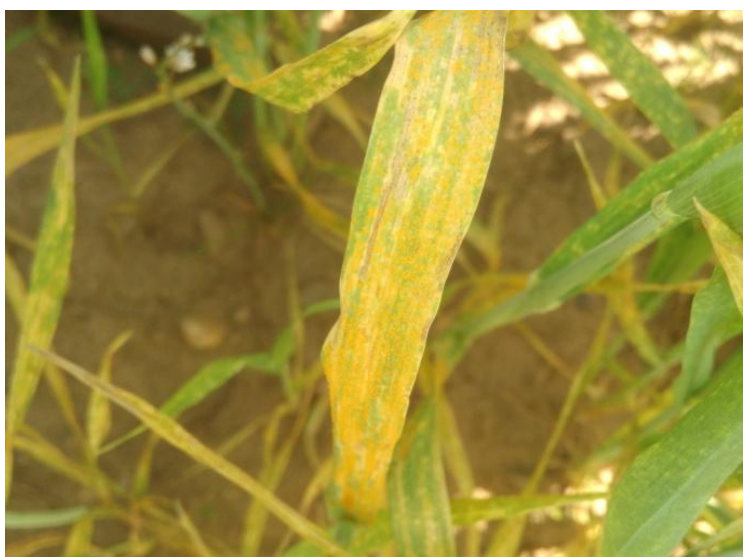

Figure 4. Attack of Puccinia striiformis

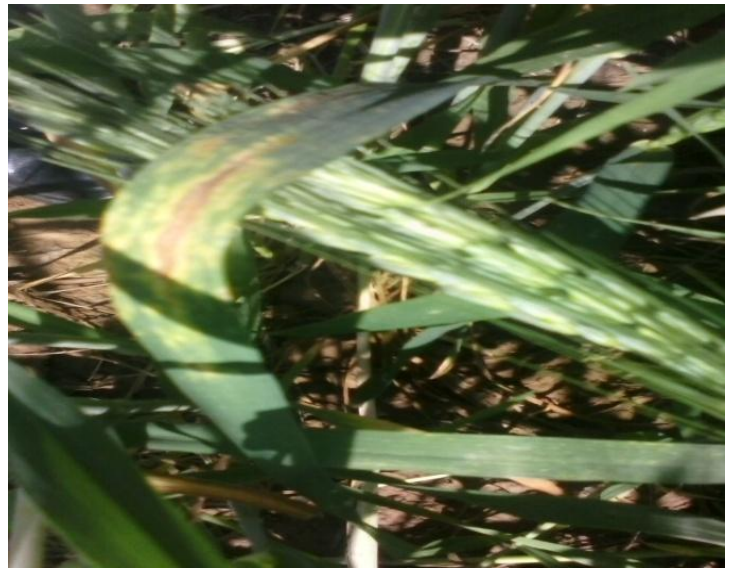

Figure 3. Attack of Stagonospora nodorum

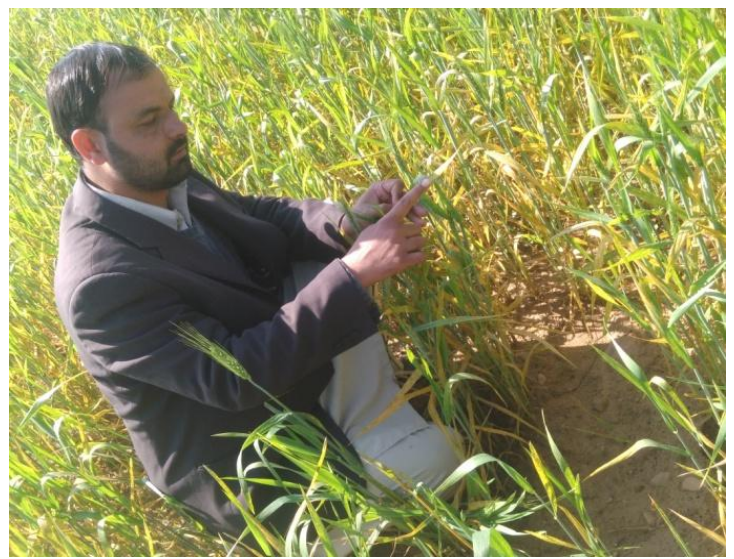

Figure 5. Attack of Drechslera tetramera 


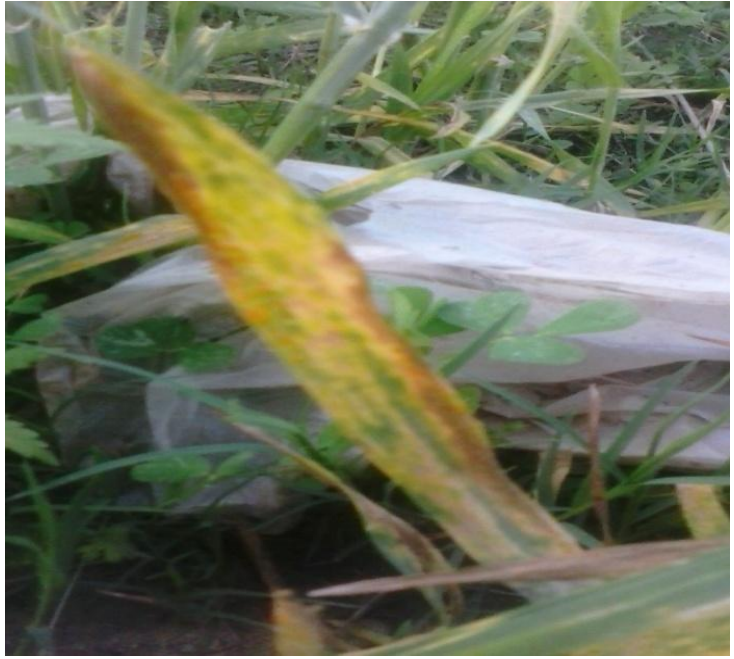

Figure 6. Multiple fungal species attack on wheat

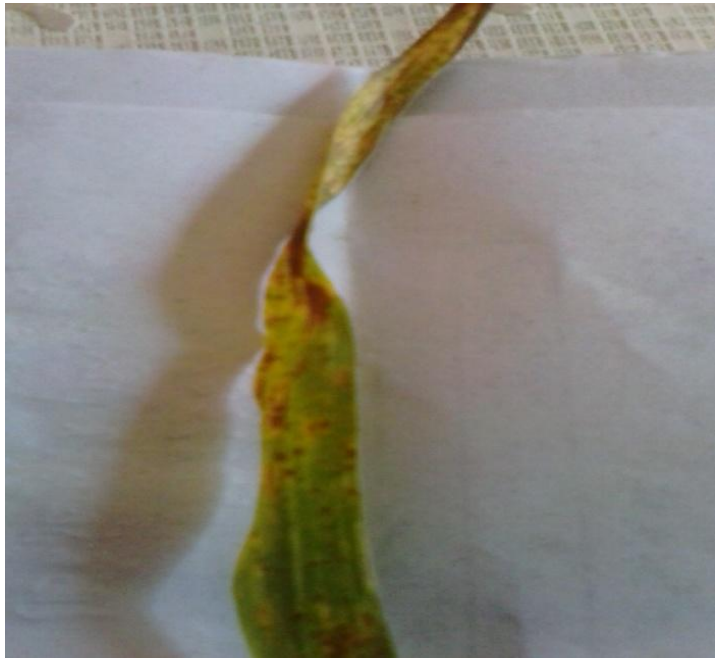

Figure 7. Attack of Alternaria species on leaf

Table 1. Survey/Isolation of Foliar mycoflora associated with eight wheat varieties from District Bhimber Azad Kashmir, Pakistan

\begin{tabular}{|c|c|c|c|c|c|c|c|c|c|c|c|}
\hline \multirow[t]{2}{*}{ S/No } & \multirow[t]{2}{*}{ Fungi detected } & \multicolumn{8}{|c|}{ Name of Wheat Varieties } & \multirow{2}{*}{$\begin{array}{c}\text { Total } \\
08\end{array}$} & \multirow{2}{*}{$\begin{array}{c}\% \\
100\end{array}$} \\
\hline & & V1 & $\mathbf{V 2}$ & V3 & V4 & V5 & V6 & V 7 & $\mathbf{V 8}$ & & \\
\hline 1 & Alternaria alternata & + & - & + & + & + & + & - & - & 5 & 62.5 \\
\hline 2 & Aspergillus niger & + & + & + & + & + & + & - & - & 6 & 75.0 \\
\hline 3 & Bipolaris sorokiniana & + & + & + & + & + & + & - & + & 7 & 87.5 \\
\hline 4 & Blumeria graminis & + & + & + & + & + & + & + & - & 7 & 87.5 \\
\hline 5 & Cephalosporium gramineum & - & + & - & + & - & + & - & - & 3 & 37.5 \\
\hline 6 & Cladosporium herbarum & + & - & + & - & + & + & - & + & 5 & 62.5 \\
\hline 7 & Drechslera tetramera & + & + & + & - & + & + & - & + & 6 & 75.0 \\
\hline 8 & D. tritici-repentis & + & - & + & - & + & + & - & - & 4 & 50.0 \\
\hline 9 & Helminthosporium sp. & + & + & + & + & + & + & + & - & 7 & 87.5 \\
\hline 10 & Microdochium nivale & + & + & + & + & + & + & - & - & 6 & 75.0 \\
\hline 11 & Mycosphaerella graminicola & + & + & + & + & + & + & + & + & 8 & 100 \\
\hline 12 & Nigrospora sphaerica & + & + & + & - & + & + & - & - & 5 & 62.5 \\
\hline 13 & Penicillium lilacinum & + & + & + & + & + & + & - & - & 6 & 75.0 \\
\hline 14 & P. chrysogenum & + & + & - & + & - & + & + & - & 5 & 62.5 \\
\hline 15 & Puccinia striiformis & + & + & + & - & + & + & - & - & 5 & 62.5 \\
\hline 16 & P. triticina & + & + & + & + & - & + & + & + & 7 & 87.5 \\
\hline 17 & $P$. recondite & + & + & + & + & + & - & + & - & 6 & 75.0 \\
\hline 18 & Pyrenophora teres & - & + & + & - & + & + & - & - & 4 & 50.0 \\
\hline 19 & P. tritici-repentis & + & + & + & - & + & + & - & - & 5 & 62.5 \\
\hline 20 & Rhizoctonia cerealis & + & - & + & - & + & - & + & + & 5 & 62.5 \\
\hline 21 & Stagonospora nodorum & + & + & + & - & + & + & + & - & 6 & 75.0 \\
\hline & Total Species Identified & 19 & 17 & 19 & 12 & 18 & 19 & 8 & 6 & 118 & \\
\hline & \%age of each variety & 90.5 & 81 & 90.5 & 57 & 85.7 & 90.5 & 38 & 28.5 & 70. & \\
\hline
\end{tabular}

Key: + = Present, - = Absent, V1= Fareed-2006, V2= Seher-2006, V3= Lasani-2008, V4= Faisalabad2008, V5= Millat-2011, V6= Aas 2011, V7= Punjab-2011, V8= Galaxy-2013 
The present mycofloral picture of District Bhimber consists of phyto-geographical snapshot of three Sub-divisions i.e., Bhimber (Bm), Samahni $(\mathrm{Sm})$ and Barnala $(\mathrm{Br})$. The prevalence of identified fungal species was calculated by Equation 1 and presented in tabular form (Table 2). The highest (100\%) prevalence of fungi was observed in three locations of $\mathrm{Sm}$, two locations of $\mathrm{Bm}$ and four locations of $\mathrm{Br}$. According to wheat varieties V1 (96\%) showed more prevalent as compared to others. While minimum prevalence was shown by V8 (70.7\%) as mentioned in Table 2.

Table 2. Prevalence (\%) of foliar diseases of eight wheat varieties from different locations of district Bhimber Azad Jammu and Kashmir, Pakistan

\begin{tabular}{llccccccccc} 
Area & Locations & \multicolumn{4}{c}{ Wheat varieties with disease prevalence (\%) } & \multicolumn{2}{c}{ Means } \\
& & V1 & V2 & V3 & V4 & V5 & V6 & V7 & V8 & \\
Samahni & 5 & 100 & 100 & 100 & 100 & 100 & 100 & 100 & 100 & 100 \\
Bandala & 5 & 100 & 100 & 100 & 100 & 100 & 100 & 100 & 100 & 100 \\
Chowki & 5 & 100 & 100 & 100 & 100 & 100 & 100 & 100 & 100 & 100 \\
Jandala & 5 & 100 & 80 & 60 & 40 & 80 & 100 & 60 & 20 & 67.5 \\
Poona & 5 & 80 & 60 & 60 & 20 & 100 & 80 & 40 & 20 & 57.5 \\
Bhimber & 5 & 100 & 60 & 60 & 80 & 80 & 60 & 40 & 40 & 65 \\
Bring & 5 & 80 & 60 & 80 & 60 & 80 & 80 & 20 & 20 & 60 \\
Sokasan & 5 & 100 & 100 & 100 & 100 & 100 & 100 & 100 & 100 & 100 \\
Punjairi & 5 & 100 & 100 & 100 & 100 & 100 & 100 & 100 & 100 & 100 \\
Kasgumma & 5 & 80 & 100 & 80 & 20 & 60 & 80 & 40 & 40 & 62.5 \\
Bernala & 5 & 100 & 100 & 100 & 100 & 100 & 100 & 100 & 100 & 100 \\
Chanb & 5 & 100 & 100 & 100 & 100 & 100 & 100 & 100 & 100 & 100 \\
Kadala & 5 & 100 & 100 & 100 & 100 & 100 & 100 & 100 & 100 & 100 \\
Tander & 5 & 100 & 80 & 80 & 60 & 100 & 80 & 20 & 20 & 67.5 \\
Thub & 5 & 100 & 100 & 100 & 100 & 100 & 100 & 100 & 100 & 100 \\
\multicolumn{1}{c}{$\%$ age of each variety } & 96 & 89 & 88.3 & 78.7 & 93.3 & 92 & 74.7 & 70.7
\end{tabular}

Key: + = Present, - = Absent, V1= Fareed-2006, V2= Seher-2006, V3= Lasani-2008, V4= Faisalabad2008, V5= Millat-2011, V6= Aas 2011, V7= Punjab-2011, V8= Galaxy-2013

Disease incidence was measured by help of Equation 3. In tehsil Samahni, wheat variety (WV) Fareed-2006 (V1) prevailed highest disease incidence (63.3\%) followed by Seher-2006 (60.3\%), Aas-2011 (59.6\%) and so on. The minimum disease incidence was measured in Galaxy-2013 (22.3\%). These results indicated that wheat variety Galaxy-2013 was more suitable for high growth and better yields production of wheat crop in the study area. This WV showed low fungal infection rate (Table 3). In tehsil Bhimber, WV Seher-2006 showed highest disease incidence $(60.0 \%)$ while minimum disease incidence was reflected in Galaxy-2013 (20.9\%). In Bernala tehsil, variety (WV) Fareed-2006 (V1) indicated highest disease incidence $(59.7 \%)$ while minimum disease incidence was measured in Galaxy-2013 (29.7\%) (Table 3).

The comparative analysis according to disease severity among three tehsils of District Bhimber was also under investigation. The Severity was measured by use of Equation 2. According to 0-9 rating scales, foliar symptoms of diseases assessed through visual basis i.e., $0=$ no symptoms, $1=10-20 \%$ spots on leaves, $2=20-30 \%$ spots, $3=30-40 \%$ spots, $4=40-50 \%$ and $5=50-60 \%$ spots, $6=60-70 \%$ spots, $7=70-80 \%$ spots, $8=80-90 \%$ spots and $9=90-100 \%$ (Table 4 ). 
Table 3. Incidence of foliar diseases of eight wheat varieties in district Bhimber Azad Kashmir

\begin{tabular}{|c|c|c|c|c|c|c|c|c|c|c|}
\hline \multirow[t]{2}{*}{ Tehseels } & \multirow[t]{2}{*}{ Locations } & \multicolumn{8}{|c|}{ *Incidence (\%) } & \multirow[t]{2}{*}{ Mean } \\
\hline & & V1 & V2 & V3 & V4 & V5 & V6 & V7 & V8 & \\
\hline \multirow[t]{6}{*}{ Samahni } & Samahni city & 81.7 & 75.0 & 78.3 & 56.7 & 78.3 & 73.3 & 46.7 & 38.3 & 66.0 \\
\hline & Bandala & 71.7 & 70.0 & 60.0 & 45.0 & 56.7 & 61.7 & 45.0 & 30.0 & 55.01 \\
\hline & Chowki & 65.0 & 58.3 & 60.0 & 40.0 & 56.7 & 56.7 & 35.0 & 21.7 & 49.2 \\
\hline & Poona & 48.3 & 46.7 & 45.0 & 20.0 & 48.3 & 48.3 & 18.3 & 8.3 & 35.4 \\
\hline & Jandala & 50.0 & 51.7 & 48.3 & 26.7 & 48.3 & 58.3 & 23.3 & 13.3 & 39.9 \\
\hline & Mean & 63.3 & 60.3 & 58.3 & 37.7 & 57.6 & 59.6 & 33.7 & 22.3 & 49.2 \\
\hline \multirow[t]{6}{*}{ Bhimber } & Bhimber city & 70 & 65.0 & 66.7 & 56.7 & 66.7 & 60.0 & 51.7 & 33.3 & 58.7 \\
\hline & Bring & 58.3 & 55.0 & 51.7 & 50.0 & 55 & 55.0 & 45.0 & 13.3 & 47.9 \\
\hline & Sokasan & 35.0 & 50.0 & 55.0 & 35.0 & 53.3 & 45.0 & 30.0 & 16.7 & 40.0 \\
\hline & Punjairi & 60.0 & 61.7 & 30.0 & 30.0 & 38.3 & 50.0 & 26.7 & 18.3 & 39.4 \\
\hline & Kasgumma & 55.0 & 68.3 & 53.3 & 33.3 & 46.7 & 48.3 & 30.0 & 23.3 & 44.8 \\
\hline & Mean & 55.7 & 60.0 & 51.3 & 41.0 & 52.0 & 51.7 & 36.7 & 20.9 & 46.2 \\
\hline \multirow[t]{6}{*}{ Barnala } & Barnala city & 76.7 & 71.7 & 73.3 & 60.0 & 71.7 & 71.7 & 53.3 & 36.7 & 64.4 \\
\hline & Chanb & 61.7 & 63.3 & 60.0 & 48.3 & 56.7 & 58.3 & 46.7 & 21.7 & 52.1 \\
\hline & Kadala & 63.3 & 55.0 & 53.3 & 36.7 & 60.0 & 53.3 & 35 & 13.3 & 46.2 \\
\hline & Tander & 48.3 & 45.0 & 40.0 & 23.3 & 26.7 & 33.3 & 18.3 & 67 & 37.7 \\
\hline & Thub & 48.3 & 50.0 & 55.0 & 35.0 & 43.3 & 43.3 & 26.7 & 10.0 & 38.95 \\
\hline & Mean & 59.7 & 57.0 & 56.3 & 40.7 & 51.7 & 51.9 & 36.0 & 29.7 & 47.8 \\
\hline
\end{tabular}

Key: *Incidence=Percentage shows wheat plants infected with foliar diseases

Table 4. Disease Severity of foliar diseases of eight wheat varieties in district Bhimber Azad Kashmir

\begin{tabular}{|c|c|c|c|c|c|c|c|c|c|c|}
\hline \multirow[t]{2}{*}{ Tehseels } & \multirow[t]{2}{*}{ locations } & \multicolumn{8}{|c|}{$*$ Severity $(0-9)$} & \multirow[t]{2}{*}{ Mean } \\
\hline & & V1 & V2 & V3 & V4 & V5 & V6 & V7 & V8 & \\
\hline \multirow[t]{6}{*}{ Samahni } & Samahni city & 8.0 & 6.3 & 7.0 & 4.3 & 6.0 & 6.0 & 4.0 & 3.0 & 5.6 \\
\hline & Bandala & 7.3 & 5.7 & 5.0 & 3.3 & 5.3 & 5.7 & 2.7 & 1.7 & 4.6 \\
\hline & Chowki & 5.0 & 4.0 & 6.0 & 4.3 & 5.7 & 5.0 & 2.7 & 3.0 & 4.5 \\
\hline & Poona & 5.0 & 3.3 & 4.0 & 4.0 & 3.7 & 3.7 & 3.3 & 1.3 & 3.5 \\
\hline & Jandala & 5.3 & 4.0 & 5.3 & 3.0 & 5.3 & 4.3 & 2.3 & 1.7 & 3.9 \\
\hline & Mean & 6.1 & 4.6 & 5.5 & 3.9 & 5.2 & 4.9 & 3.0 & 2.1 & 4.4 \\
\hline \multirow[t]{6}{*}{ Bhimber } & Bhimber city & 8.0 & 6.0 & 6.0 & 3.7 & 5.0 & 6.0 & 4.0 & 2.7 & 5.2 \\
\hline & Bring & 7.3 & 5.3 & 5.0 & 3.0 & 4.7 & 5.3 & 2.3 & 1.0 & 4.2 \\
\hline & Sokasan & 6.7 & 6.3 & 6.7 & 4.7 & 5.3 & 4.0 & 2.3 & 2.0 & 4.7 \\
\hline & Punjairi & 4.7 & 5.3 & 7.7 & 2.3 & 3.0 & 3.7 & 3.0 & 1.0 & 3.8 \\
\hline & Kasgumma & 6.0 & 7.0 & 6.0 & 2.0 & 4.0 & 5.7 & 2.0 & 1.3 & 4.3 \\
\hline & Mean & 6.5 & 5.9 & 6.3 & 3.1 & 4.4 & 4.9 & 2.7 & 1.6 & 4.4 \\
\hline \multirow[t]{6}{*}{ Barnala } & Bernala city & 7.7 & 7.0 & 7.7 & 4.7 & 6.3 & 6.3 & 4.0 & 2.7 & 5.8 \\
\hline & Chanb & 6.7 & 6.7 & 6.0 & 4.0 & 4.7 & 5.3 & 2.3 & 2.0 & 4.7 \\
\hline & Kadala & 5.0 & 7.7 & 6.0 & 4.0 & 6.7 & 5.3 & 3.0 & 3.0 & 5.1 \\
\hline & Tander & 5.3 & 4.0 & 3.3 & 1.7 & 3.3 & 3.3 & 1.7 & 1.7 & 3.0 \\
\hline & Thub & 7.3 & 7.0 & 5.0 & 2.7 & 5.7 & 4.7 & 2.7 & 1.3 & 4.5 \\
\hline & Mean & 6.4 & 6.5 & 5.6 & 3.4 & 5.3 & 4.9 & 2.7 & 2.1 & 4.6 \\
\hline
\end{tabular}

*Severity $=0-9$ rating scales show foliar symptoms of diseases assessed through visual basis i.e., $0=$ no symptoms, $1=10-20 \%$ spots on leaves, $2=20-30 \%$ spots, $3=30-40 \%$ spots, $4=40-50 \%$ and $5=50-60 \%$ spots, $6=60-70 \%$ spots, $7=70-80 \%$ spots, $8=80-90 \%$ spots, $9=90-100 \%$

In tehsil Samahni, highest severity was shown by WV Fareed-2006 (V1) as 6.1 on severity scale. The minimum severity was shown by Galaxy-2013 (2.1). Less severity on wheat variety produced better yields. In tehsil Bhimber, variety (WV) Seher-2006 
(V1) indicated highest disease severity (6.5) while minimum disease severity was measured in Galaxy-2013 (1.6). In Barnala tehsil, highest severity was shown by V1 and V2 $(6.4,6.5)$ while minimum disease severity was seen in Galaxy-2013 (2.1) as indicated in Table 4.

\section{Management strategies}

Foliar diseases of WV were managed by applying fungicides treatments through chemical spray and biological treatments by different plant extracts spray. In this research, five different fungicides were used for chemical treatment to wheat leaves at early growth stages/before appearance of fungal disease symptoms. These fungicides reduced fungal infections and improved wheat yields.

The total yields of eight WV were measured before and after treatment for comparative analysis. Without treatments, highest yield was obtained from V8 (1543 kg $\left.\mathrm{ha}^{-1}\right)$. On the other hand, low yield was received from V1 (1210 kg ha $\left.{ }^{-1}\right)$ and V2 (1245 $\mathrm{kg} \mathrm{ha}^{-1}$ ) as indicated in Table 5. After fungicides spray highest yield was obtained from V8 (1706 kg ha $\left.{ }^{-1}\right)$ which is better than untreated samples. Similarly, $1602 \mathrm{~kg} \mathrm{ha}^{-1}$ yield was produced by V4 while lowest yield was received from V1 $\left(1362 \mathrm{~kg} \mathrm{ha}^{-1}\right)$ that is also better from untreated samples as mentioned above (Table 6 ).

Table 5. Total yields of untreated field trials of different wheat varieties in district Bhimber Azad Kashmir, Pakistan

\begin{tabular}{|c|c|c|c|c|c|c|c|c|c|c|}
\hline \multirow{2}{*}{ S/No } & \multirow{2}{*}{$\begin{array}{c}\text { Field } \\
\text { Trails }\end{array}$} & \multicolumn{8}{|c|}{ Wheat Varieties with their yield $\left(\mathrm{kg} \mathrm{ha}^{-1}\right)$ before fungicide treatment } & \multirow[t]{2}{*}{ Means } \\
\hline & & V1 & V2 & V3 & V4 & V5 & V6 & V7 & $\mathbf{V 8}$ & \\
\hline 1 & $\mathrm{~T} 1$ & 1150 & 1260 & 1300 & 1460 & 1430 & 1340 & 1480 & 1460 & $1360^{\mathrm{a}}$ \\
\hline 2 & $\mathrm{~T} 2$ & 1200 & 1280 & 1250 & 1390 & 1340 & 1270 & 1420 & 1489 & $1330^{a b}$ \\
\hline 3 & T3 & 1220 & 1190 & 1360 & 1450 & 1210 & 1340 & 1390 & 1570 & $1341^{\mathrm{a}}$ \\
\hline 4 & $\mathrm{~T} 4$ & 1180 & 1298 & 1230 & 1270 & 1195 & 1290 & 1550 & 1620 & $1329^{a b}$ \\
\hline 5 & T5 & 1300 & 1200 & 1175 & 1520 & 1290 & 1380 & 1500 & 1580 & $1368^{\mathrm{a}}$ \\
\hline \multicolumn{2}{|c|}{ Average } & $1210^{\mathrm{e}}$ & $1245^{\mathrm{e}}$ & $1263^{d}$ & $1418^{b}$ & $1293^{d}$ & $1324^{c}$ & $1468^{b}$ & $1543^{a}$ & \\
\hline
\end{tabular}

Key: $\mathrm{T} 1=$ Trail $1, \mathrm{~T} 2=$ Trail $2, \mathrm{~T} 3=$ Trail $3, \mathrm{~T} 4=$ Trail $4, \mathrm{~T} 5=$ Trail 5

Table 6. Fungicides treatment on leaves of wheat varieties and measured total yield increase ( $\mathrm{kg} \mathrm{ha}^{-1}$ ) from Bhimber district Azad Jammu and Kashmir, Pakistan

\begin{tabular}{|c|c|c|c|c|c|c|c|c|c|c|}
\hline \multirow{2}{*}{ S/No } & \multirow{2}{*}{ Fungicide } & \multicolumn{8}{|c|}{ Wheat Varieties with yield increase $\left(\mathrm{kg} \mathrm{ha}^{-1}\right)$ after fungicides treatment } & \multirow[t]{2}{*}{ Means } \\
\hline & & V1 & V2 & V3 & V4 & V5 & V6 & V7 & V8 & \\
\hline 1 & F1 & 1300 & 1380 & 1490 & 1600 & 1580 & 1440 & 1610 & 1690 & $1511^{\mathrm{b}}$ \\
\hline 2 & $\mathrm{~F} 2$ & 1350 & 1560 & 1385 & 1580 & 1500 & 1390 & 1580 & 1700 & $1506^{b}$ \\
\hline 3 & F3 & 1410 & 1480 & 1520 & 1450 & 1450 & 1460 & 1550 & 1680 & $1500^{\mathrm{ab}}$ \\
\hline 4 & F4 & 1290 & 1500 & 1500 & 1685 & 1470 & 1400 & 1745 & 1810 & $1550^{a}$ \\
\hline 5 & F5 & 1460 & 1520 & 1460 & 1695 & 1495 & 1530 & 1670 & 1650 & $1560^{a}$ \\
\hline & verage & $1362^{e}$ & $1488^{c}$ & $1471^{c}$ & $1602^{a b}$ & $1499^{c}$ & $1444^{\mathrm{cd}}$ & $1631^{b}$ & $1706^{a}$ & \\
\hline
\end{tabular}

Key: F1 = Tilt (0.29 1 ha-1), F2= Proline (0.76 1 ha-1), F3= Strobilurins (0.89 1 ha-1), F4= Quadris $(0.581$ ha-1), F5= Headline (0.58 1 ha- 1$)$

The impact of fungicides on yield rate of wheat varieties were also calculated and documented in Table 6. After spray of fungicide Quadris (0.58 1 ha-1) and Headline 
(0.58 1 ha-1) on wheat trails, maximum yield was produced as compared to other fungicides i.e., $1550 \mathrm{~kg} \mathrm{ha}^{-1}, 1560 \mathrm{~kg} \mathrm{ha}^{-1}$. This means that the fungicide F4 and F5 are more effective to combat and reduce fungal diseases from surfaces of wheat leaves (Table 6).

On the other hand, after different plant extracts treatment/spray on wheat variety trails was showed maximum yields was obtained from V8 $\left(1739 \mathrm{~kg} \mathrm{ha}^{-1}\right)$ as compared to others WV. Lowest yield was obtained by WV Seher-2006 (1348 kg ha' $)$ as mentioned in Table 7.

Table 7. Treatment of leaves of wheat varieties with different crude plant extracts in Bhimber district Azad Jammu and Kashmir, Pakistan

\begin{tabular}{|c|c|c|c|c|c|c|c|c|c|c|}
\hline S/ & & & Wheat v & ieties $\mathbf{s}$ & red wit & differen & plants c & ide extr & & Mogn \\
\hline $\mathbf{N}$ & Plant Name & V1 & V2 & V3 & V4 & V5 & V6 & V7 & V8 & vitean \\
\hline 1 & Acacia nilotica & 1350 & 1400 & 1490 & 1590 & 1570 & 1540 & 1650 & 1680 & $1534^{\mathrm{ab}}$ \\
\hline & $\begin{array}{l}\text { Azadirachta } \\
\quad \text { indica }\end{array}$ & 1330 & 1500 & 1370 & 1570 & 1560 & 1400 & 1590 & 1750 & $1509^{b}$ \\
\hline & $\begin{array}{l}\text { Eucaylptus } \\
\text { citriodora }\end{array}$ & 1420 & 1450 & 1550 & 1490 & 1400 & 1450 & 1565 & 1685 & $1501^{b}$ \\
\hline & $\begin{array}{c}\text { Ficus } \\
\text { bengalensis }\end{array}$ & 1250 & 1470 & 1580 & 1630 & 1480 & 1460 & 1770 & 1870 & $1564^{\mathrm{a}}$ \\
\hline & Curcuma longa & 1390 & 1570 & 1420 & 1590 & 1500 & 1580 & 1685 & 1710 & $1556^{\mathrm{a}}$ \\
\hline & Average & $1348^{\mathrm{f}}$ & $1478^{\mathrm{e}}$ & $1482^{\mathrm{e}}$ & $1574^{\mathrm{c}}$ & $1502^{\mathrm{d}}$ & $1486^{\mathrm{e}}$ & $1652^{b}$ & $1739^{a}$ & \\
\hline
\end{tabular}

\section{Discussion}

Wheat (Triticum aestivum) is suffered from many diseases some of them are foliar pathogens. In this current research, 21 different foliar fungal species were identified from eight wheat varieties. The foliar samples of eight wheat varieties were collected and brought to Laboratory of Botany, Mirpur University of Science and Technology (MUST), Bhimber Campus Azad Jammu and Kashmir for experimental works.

Out of 21 fungal species $19(90.5 \%)$ were isolated from V1 (Fareed-2006), V3 (Lasani-2008) and V6 (Aas-2011). these Highest number of fungal species were depicted that the ecoclimatic conditions of district Bhimber were more suitable for fungal growth and reproduction during wheat seasons. Secondly, these three wheat varieties have less resistance against fungal pathogens. Therefore fungal spores were easily attack on leaves of the varieties (Arregui and Puricelli, 2008). Low fungal frequencies (6 species) were identified from wheat variety V8 (Galaxy-2013). This means that V8 variety is more susceptible to fungi and more disease resistance as compared to other WV. Hence, it is more suitable for sowing in the study area. Similar observations were analyzed by Ortiz et al. (2008).

In current analysis, fungal pathogen Mycosphaerella graminicola was isolated from all wheat varieties. This may be due to high moisture contents and optimum temperature in District Bhimber Azad Kashmir. As previously same climatic study was conducted by Wagacha and Muthomi (2007). The fungi Cephalosporium gramineum was only present on three wheat varieties with $37.5 \%$ as indicated in Table 1. This fungal species did not spread more in the study area due to unfavorable environmental conditions. 
The present mycofloral picture of District Bhimber consists of a comparative study of phyto-geographical diversity and comparative mycological analysis of three subdivisions (tehsils) of district Bhimber Azad Kashmir, i.e. Bhimber (Bm), Samahni (Sm) and Barnala (Br). Similar prevalence of fungi in the three areas depicts their ubiquity in all ecosystems of this climate. According to wheat varieties V1 is more vulnerable to fungal diseases while minimum dominancy was shown by V8 as mentioned in Table 2. These findings were similar to above described results as discussed previously by Singh et al., (1998).

These results indicated that wheat variety Galaxy-2013 was more suitable for high growth and better yields production of wheat crop in the study area. This WV showed low fungal infection rate and fewer incidences due to better and quicker adaptation in the area (Sharma and Duveiller, 2003).

The comparative analysis according to disease severity among the three subdivisions of district Bhimber was also carried out. The Severity was measured according to 0-9 rating scales which showed foliar symptoms of diseases assessed through visual basis i.e., $0=$ no symptoms, $1=10-20 \%$ spots on leaves, $2=20-30 \%$ spots, $3=30-40 \%$ spots, $4=40-50 \%$ and $5=50-60 \%$ spots, $6=60-70 \%$ spots, $7=70-80 \%$ spots, $8=80-90 \%$ spots and 9=90-100\% (Bockus et al., 2010). Highest severity was calculated from WV Fareed-2006 (V1) on severity scale. This is also due to low resistance. Secondly, it may be due to repetition every year in the study area. Therefore, it becomes more vulnerable to fungi (Chakraborty et al., 2011).

The severity was also minimum on Galaxy-2013. Less severity on wheat variety produced good yields. Similar findings were obtained in other locations. This means that overall results can be explained by that WV Galaxy-2013 was first time introduced in the study area. Therefore, it was less effective less as compared to the other seven WVs. Therefore former should be preferred to this variety in the study area for better food and fodder yields (Fernandes et al., 2004).

\section{Management strategies}

The population on this planet increases day by day and agricultural lands have been reduced. This is an alarming horizon for future. Demand for food is increasing day by day. Therefore we should carefully think on this issue: what is a good way to increase food and fodder yields? We should control foliar diseases of WV by applying fungicides treatments through chemical spray and biological spray treatment of different plant extracts. In this research, five different fungicides were used as chemical treatment on wheat leaves at appearance of fungal disease symptoms. Spray of fungicides reduced fungal infections especially and improved wheat yields (Varga et al., 2005; Carmona et al., 1999).

The total yields of eight WVs were measured before and after treatment for comparative analysis. Without treatments low yield was received from V1 (1210 kg ha $\left.{ }^{-1}\right)$ and V2 (1245 kg ha-1) as indicated in Table 5. After fungicides spray the highest yield was obtained fromV8 which was better than untreated samples. Similar research was conducted by Gooding (2006).

The impact of fungicides on yield rate of wheat varieties were also calculated and documented. After spray of fungicides on wheat trails, maximum yield was produced as compared to other fungicides. This means that the fungicides F4 and F5 are more effective to combat and reduce fungal diseases from surfaces of wheat leaves (Oerke, 2006; Ortiz et al., 2008). 
On the other hand, after different plants extracts (Acacia nilotica, Azadirachta indica, Curcuma longa, Eucaylptus citriodora, Ficus bengalensis) treatment on wheat variety trails good yields were produced. This means that after biocontrol greater yields of wheat was obtained according to requirements. Similar biocontrol was applied by Benkeblia (2004). Finally, biological treatments are recommended to WV after sowing because this technique does not have fatal impact on health and environments. Similar work was done by Aqil et al., (2010). This biological method is cheap and easily manageable compared to every former. Previously, similar biological treatments were launched by Iftikhar et al., (2012) and Pathak and Razia (2013).

Fungicides treatments also resulted more net yields but they polluted the environment, produced harmful impacts on flora and fauna. Secondly, they also were costly applications. Therefore, we should prefer this new technique of plant extracts for fungal foliar disease management.

\section{Conclusion}

From these findings it is concluded that the control measures of fungal pathogens is a basic need to avoid crop failure. It is desirable that after sowing wheat crop, spreading of pathogens in wheat growing areas are treated with fungicides and biological treatments to attain maximum yield of wheat crops. In current research attention has been given to use biological techniques for foliar treatment to protect them against fungal pathogens. Chemical fungicides can control the crop diseases, but they have bad effects on plants, animals and human health and also on our environment. Since these fungicides are likely to have hazardous impacts on human life and environment, it is therefore necessary to search for better control measures that are cheap, ecologically sound and environmentally safe to eliminate or reduce the incidence of these pathogens and for the improvement of wheat quality and quantity, so as to obtain healthy and strong wheat plants for fodder as well as better yields of different varieties of wheat crop. Therefore biological treatment of wheat fungal diseases are recommended for obtaining better yields of wheat crop in future in the examined area.

\section{REFERENCES}

[1] Arregui, M.C., Puricelli, E. (2008): Mecanismos y modo de acción de fungicidas.- Dow Agroscience 208 .

[2] Aqil, F., Zahin, M., Ahmed, I., Owais, M., Khan, M.S.A, Bansal, S.S., Farooq, S. (2010): Antifungal Activity of Medical Plant Extracts and Phytocompounds: A Review - In: Ahmad, I., Owais, M., Shahid, M. (eds.) Combating Fungal Infections: Problem and Remedy, Springer-Verlag, Berlin, Heidelberg, Germany, pp. 449-485.

[3] Bender, J., Weigel, H. J. (2011): Changes in atmospheric chemistry and crop health: a review-Agronomy for Sustainable Development 31:81-89.

[4] Bockus, W.W., Bowden, R.L., Hunger, R.M., Morrill, W.C., Murray, T.D., Smiley, R.W. (2010): Compendium of wheat diseases and pests. - St. Paul: APS Press.

[5] Brown, K.H., Wuehler, S.E., Peerson, J.M. (2001): The importance of zinc in human nutrition and estimation of the global prevalence of zinc deficiency.- Food Nutr. Bull. 22:113-125.

[6] Benkeblia, N. (2004): Antimicrobial activity of essential oil extracts of various onions (Allium cepa) and garlic (Allium sativum)- LWT- Food Sci. Technol. 37 (2): 263-268. 
[7] Carmona, M., Cortese. P., Moschini, R., Pioli, R., Ferrazini, M., Reis, E. (1999): Economical damage threshold for fungicide control of leaf blotch and tan spot of wheat in Argentina.- XIV th International Plant Protection Congress, Jerusalem, Israel, 25-30 July 1999:119.

[8] Chakraborty, S., Luck, J., Hollaway, G., Fitzgerald, G., White, N. (2011): Rust-proofing wheat for a changing climate.- Euphytica 179:19-32.

[9] Chaurasia. P.C.P., Duveiller, E. (2006): Management of leaf blight (Bipolaris sorokiniana) disease of wheat with cultural practices.- Nepal Agric. Res. J. 7:63-69.

[10] Champeil, A., Dor'e, T., Fourbet, J.F. (2004): "Fusarium head blight: Epidemiological origin of the effects of cultural practices on head blight attacks and the production of mycotoxins by Fusarium in wheat grains.- Plant Science 166(6): 1389-1415.

[11] Carignano, M., Staggenborg, S.A., Shroyer, J.P. (2008): Management practices to minimize tan spot in a continuous wheat rotation.- Agronomy Journal. 100:145-153.

[12] Domsch, K.H., Gams, W., Anderson, T.H. (1980): Compendium of soil fungi.- Academic press. A subsidiary of Harcourt brace

[13] Dubin, H.J., Bimb, H.P. (1994): Studies of soil borne diseases and foliar blights of wheat at the National Wheat Research Experiment Station, Bhairahawa, Nepal.- Wheat special report no 36. CIMMYT, Mexico.

[14] Dumalasova, V., Bartos, P. (2009): Will climatic changes enhance the risk of Tilletia indica in Europe? - Plant Protection Science 45(Special Issue). 38-40.

[15] Ewert, F. (2012): Adaptation: opportunities in climate change?- Nature Climate Change 2: $153-154$.

[16] FAO (2012). Food and Agriculture Organisation of the United Nations,- FAOSTAT, FAO Statistics Division.

[17] Fazal, M.J.R, Ihsan, I. (2005): Callus Induction and Regeneration in Seed Explants of Rice (ORYZA SATIVA CV.SWAT.2).- Pakikistan Journal of Botany (37): 829-836.

[18] Fernandes, J.M., Cunha, G.R., Del P.E., Pavan, W., Pires, J.L., Baethgen, W., (2004): Modeling fusarium ear blight in wheat under climate change using linked process-based models. - Proceedings of the 2nd International Symposium on Fusarium Head Blight, incorporating the 8th European FusariumSeminar, 11-15 December 2004, Orlando, pp. 441-443.

[19] Gilaman, J.C. (1945): A manual of soil fungi.- The Iowa State University Press, Ames, Iowa, U.S.A.

[20] Gooding, M.J. (2006): The effect of fungicides on the grain yield and quality of wheat.Actas del Congreso "A Todo Trigo". 18 y 19 de Mayo de 2006, Mar del Plata, Argentina, pp. 45-52.

[21] Hussain, T., Ishtiaq, M., Hussain A., Sultana, K. (2011): Study of Drinking Water Fungi and its Pathogenic Effects on Human Beings From District Bhimber Azad Kashmir, Pakistan.-Pak. J. Bot. 43(5): 2581-2585.

[22] Iftikhar, S., Asad, S., Rattu, A., Anjum, M., Fayyaz, M. (2012): Screening of commercial wheat varieties to spot blotch under controlled and field conditions. - Pak. J. Bot. 44:361363.

[23] Juroszek, P., Tiedemann, A. (2011): Potential strategies and future requirements for plant disease management under a changing climate.- Plant Pathology 60:100-112.

[24] Jaczewska, K.A. (2010): Efekt ekonomiczny zwalczania chorób grzybowych pszenicy ozimej latach.- Swiat Zbó. 14: 16-19.

[25] Korbas, M. (2004): Choroby podstawy zdzbła -mofliwosci i perspektywy zwalczania.Plant Protection 44: 147-154.

[26] Kaur, S., Dhaliwal, L., Kaur, P. (2008): Impact of climate change on wheat disease scenario in Punjab.- Journal of Research 45:161-170.

[27] Madgwick, J.W., West, J.S., White, R.P., Semenov, M.A., Townsend, J.A., Turner, J.A. (2011): Impacts of climate change on wheat anthesis and fusarium ear blight in the UK.European Journal of Plant Pathology 130: 117-131. 
[28] Ortiz, R., Sayre, K. D., Govaerts, B., Gupta, R., Subbarao, G.V., Ban, T. (2008): Climate change: can wheat beat the heat?- Agriculture, Ecosystems \& Environment 126: 46-58.

[29] Oerke, E.C. (2006): Crop losses to pests.- The Journal of Agricultural Science 144: 3143.

[30] Pathak, N., Razia, K.Z. (2013): Comparative study of seed dressing fungicides and Calotropis procera latex for the control of seed-borne mycoflora of wheat.- Annals of Biological Research 4 (4):1-6.

[31] Pettitt, T.R., Parry, D.W. (1996): Effects of climate change on Fusarium foot rot of winter wheat in the United Kingdom.- In: Frankland, J. C., Magan, N., Gadd, G. M. (eds.) Fungi and environmental change., Cambridge: Cambridge University Press, pp. 20-31.

[32] Rosada, J., Dubas, A., Bubniewicz, P. (2010): Perspektywy agrotechnicznych metodochrony roslin.- Progress in Plant Protection 50: 1181-1187.

[33] Roth, G.W., Marshall, H.G. (1987): Effects of timing of nitrogen fertilization and a fungicide on soft red winter wheat.- Agronomy Journal 79:197-200.

[34] Sharma, R.C., Duveiller, E. (2003): Effect of stress on Helminthosporium leaf blight in wheat.- In: Rasmussen, J.B., Friesen, T. L., Ali, S. (eds) Proc. 4th int. wheat tan spot and spot blotch workshop, North Dakota State University, Fargo, pp. 140-144.

[35] Sharma, R.C., Duveiller, E. (2004): Effect of Helminthosporium leaf blight on performance of timely seeded wheat under optimal and stress levels of soil fertility and moisture.- Field Crop Research 89:205-218.

[36] Singh, R.V., Singh, A.K., Ahmad, R., Singh, S.P. (1998): Influence of agronomic practices on foliar blight and identification of alternate hosts in the rice-wheat cropping systems.-In: Duveiller, E., Dubin, H.J., Reeves, J., McNab, A. (eds) Helminthosporium leaf blight of wheat: Spot blotch and tan spot, CIMMYT, Mexico, pp. 346-348.

[37] Steel, R.G.D., Torrie, J.H., Dickey, D. (1996): Principles and Procedures of Statistics: A Biometrical Approach, $3^{\text {rd }}$ edition. - Mc Graw-Hill, New York, U.S.A.

[38] Teng, P.S., James, W.C. (2001): Disease and yield loss assessment.- In: Waller, J.M., Lenne, J.M., Waller, S.J. (eds.): Plant Pathologist's Pocketbook, CABI Publishing Company Inc., Boston, Massachussetts, pp. 25-38.

[39] Usmani, S.M.H., Ghaffar, A. (1982): Polyethylene mulching of soil to reduce viability of sclerotia of Sclerotium oryzae.- Soil Biol. Biochem. 14: 203-206.

[40] Varga, B., Svecnjak, Z., Macesic, D., Uher, D. (2005): Winter wheat cultivar responses to fungicide application are affected by nitrogen fertilization rate.- Journal of Agronomy and Crop Science 191: 130-137.

[41] West, J.S., Holdgate, S., Townsend, J.A., Edwards, S.G., Jennings, P., Fitt, B.D.L. (2012): Impacts of changing climate and agronomic factors on fusarium ear blight of wheat in the UK.- Fungal Ecology 5: 53-61.

[42] Wagacha, J.M., Muthomi, J.W. (2007): Fusarium culmorum: Infection process, mechanisms of mycotoxin production and their role in pathogenesis in wheat.- Crop Protection 26: 877-885.

[43] Wolf, A., Schramm, U., Fahr, A., Aicher, L., Cordier, A., Trommer, W.E., Fricker, G. (1998): Hepatocellular effects of cyclosporine A and its derivative SDZ IMM 125 in vitro.- J. Pharmacol. Exp. Ther. 284: 817-825. 


\section{APPENDIX}

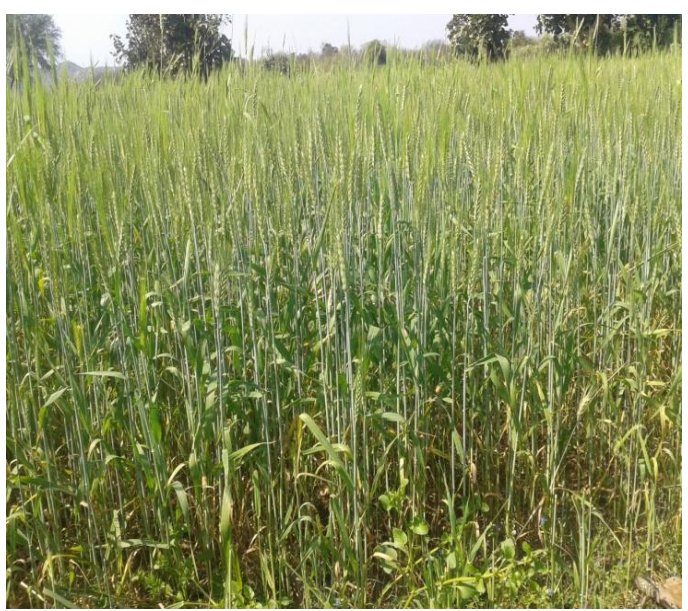

Appendix 1. Wheat variety Fareed-2006 (V1)

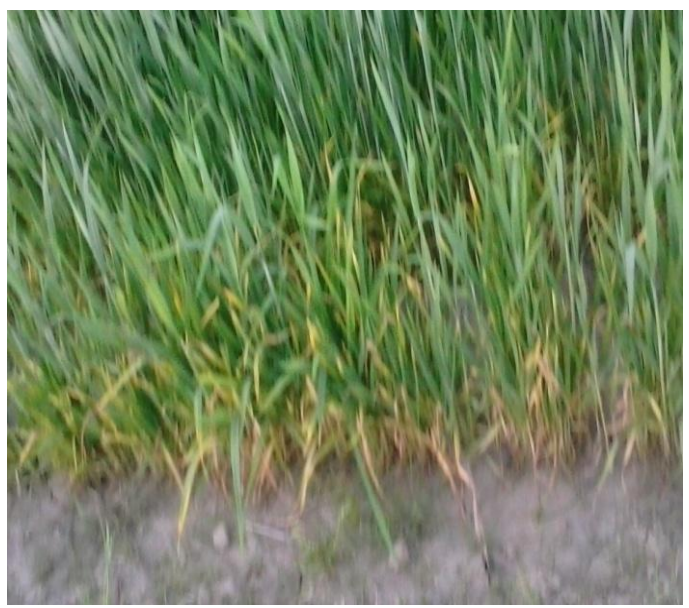

Appendix 2. Wheat variety Seher-2006 (V2)

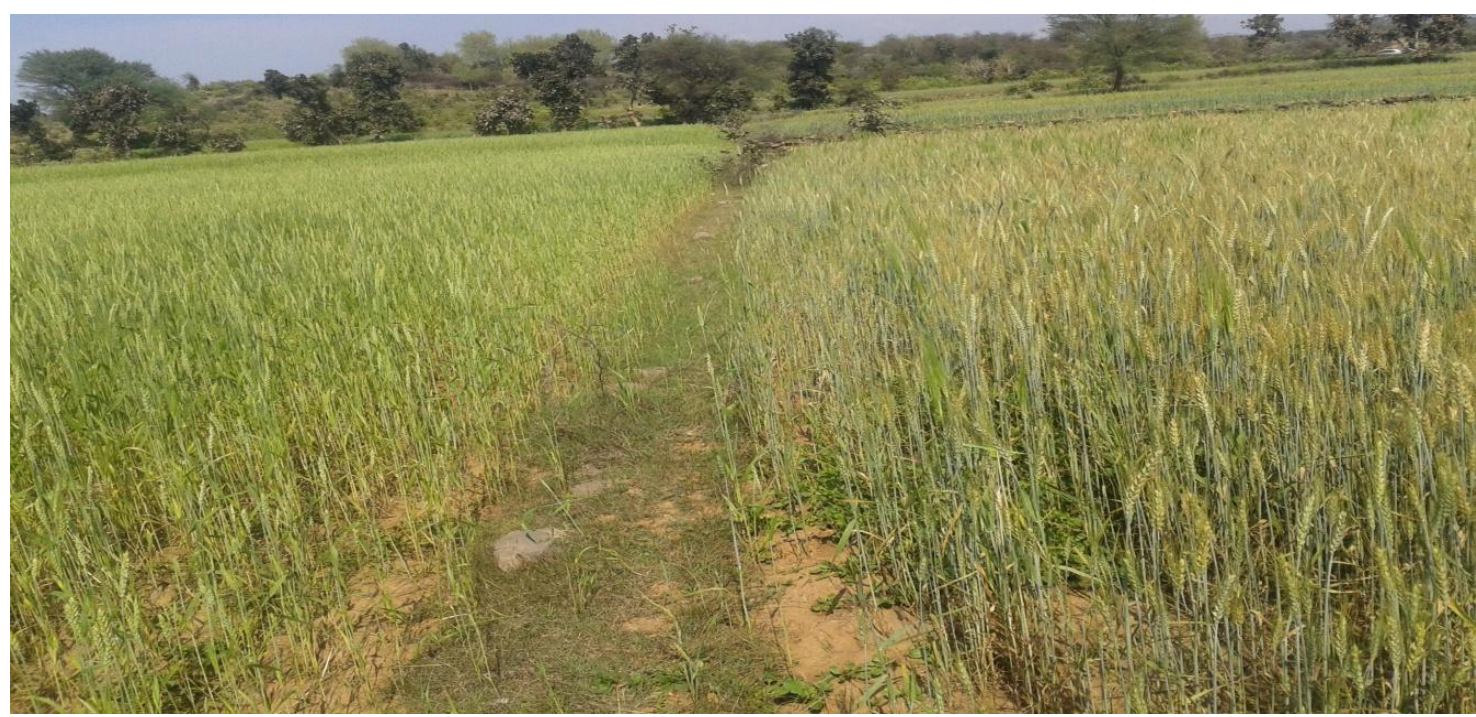

Appendix 3. Wheat variety Lasani-2008 (V3), Wheat variety Faisalabad- 2008 (V4)

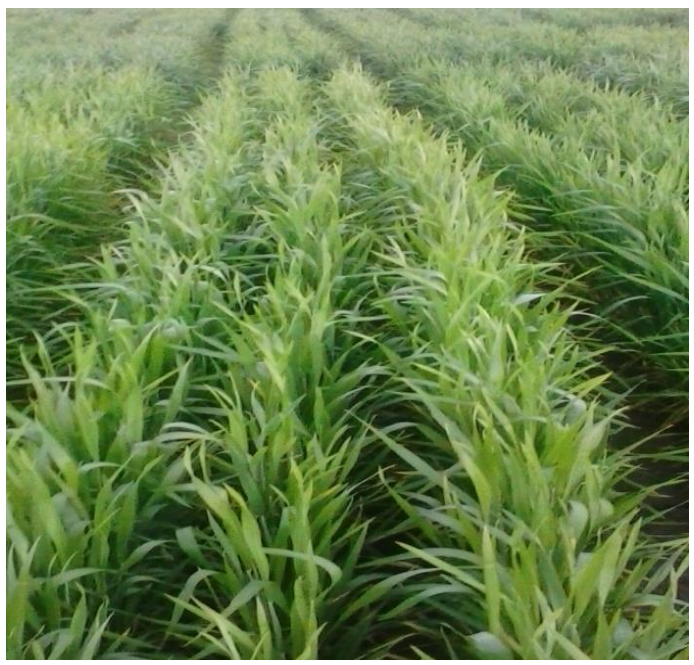

Appendix 4. Wheat variety Millat-2011(V5)

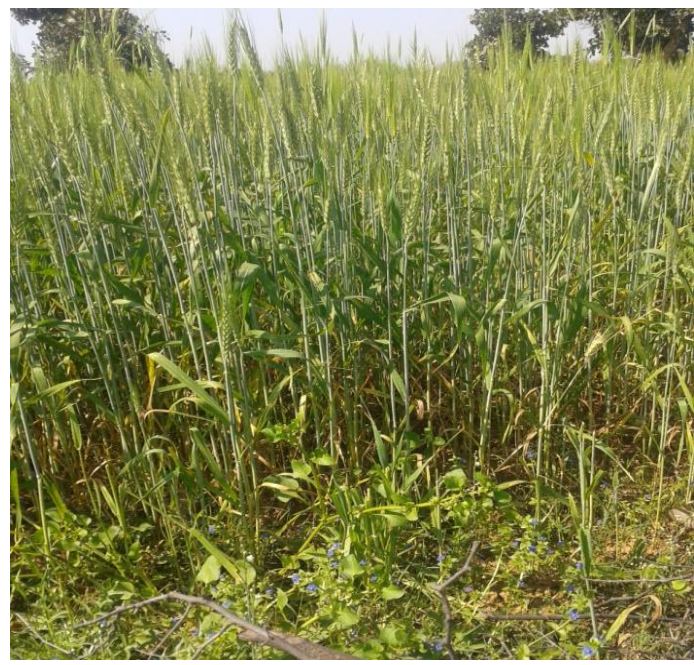

Appendix 5. Wheat variety Aas 2011 (V6) 


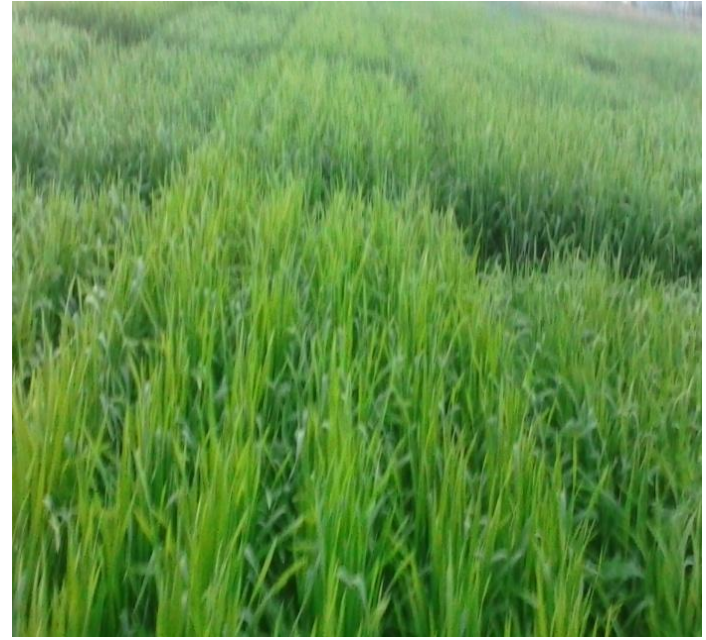

Appendix 6. Wheat variety Punjab-2011 (V7)

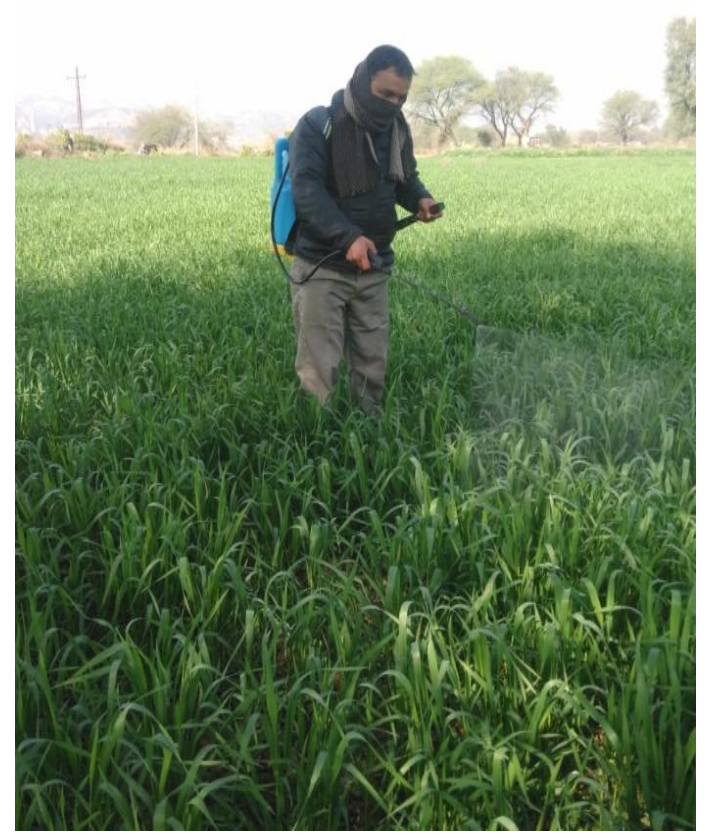

Appendix 8. Spray of Fungicides in field trails

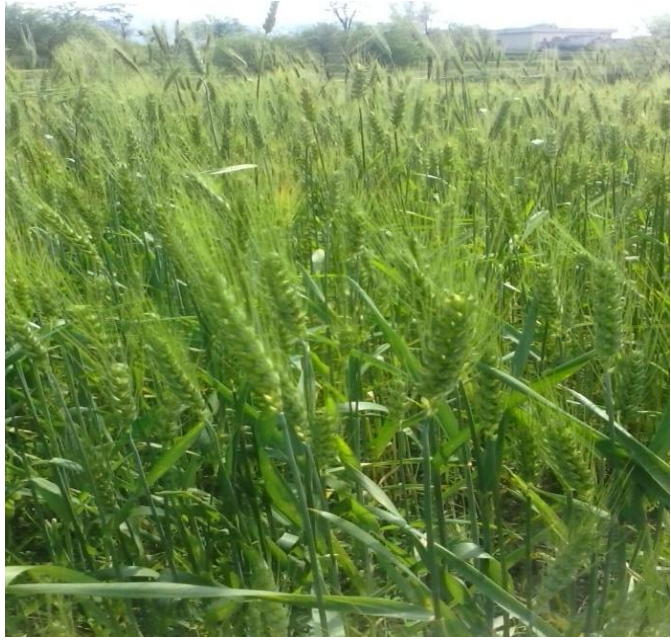

Appendix 7. Wheat variety Galaxy-2013 (V8)

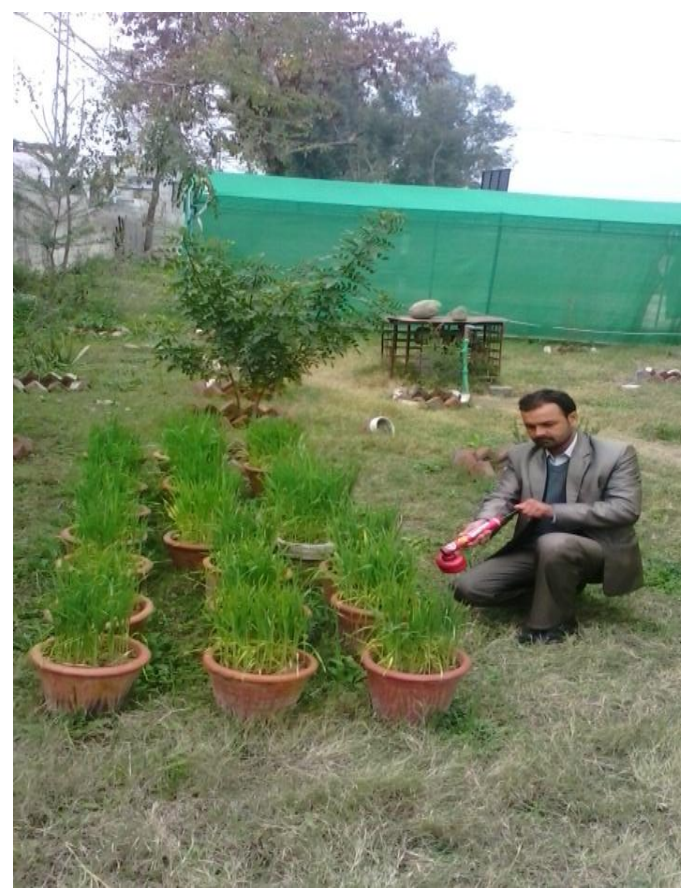

Appendix 9. Spray of Plants extracts on wheat trails 